\title{
AUTHOR INDEX VOLUME 23 (2013)
}

Acharya, U. R., see Martis, R. J.

4 (2013) 1350014

Acharya, U. R., see Martis, R. J.

5 (2013) 1350023

Acharya, U. R., Yanti, R., Wei, Z. J., Krishnan, M. M. R., Hong, T. J., Martis, R. J. \&

3 (2013) 1350009

Min, L. C., Automated diagnosis of epilepsy using CWT, HOS and texture parameters

Achten, R., see Vonck, K.

Alexandridis, A., Evolving RBF neural networks for adaptive soft-sensor design

Álvarez, D., Hornero, R., Marcos, J. V., Wessel, N., Penzel, T., Glos, M. \& del Campo,

F., Assessment of feature selection and classification approaches to enhance information from overnight oximetry in the context of apnea diagnosis

Amina, M., see Kodogiannis, V. S.

Andreou, A. G., see Dura-Bernal, S.

Astikainen, P., see Cong, F.

Badawy, R. A. B., Jackson, G. D., Berkovic, S. F. \& Macdonell, R. A. L., Cortical excitability and refractory epilepsy: A three-year longitudinal transcranial magnetic stimulation study

Bardakjian, B. L., see Serletis, D.

Berkovic, S. F., see Badawy, R. A. B.

Boon, P., see Mollet, L.

Boon, P., see Vonck, K.

Boutalis, Y., Christodoulou, M. \& Theodoridis, D., Indirect adaptive control of nonlinear systems based on bilinear neuro-fuzzy approximation

Boylan, G., see Temko, A.

Cappaert, N. L. M., Ramekers, D., Martens, H. C. F. \& Wadman, W. J., Efficacy of a new charge-balanced biphasic electrical stimulus in the isolated sciatic nerve and the hippocampal slice

1 (2013) 1250034

6 (2013) 1350029

5 (2013) 1350020

5 (2013) 1350024

5 (2013) 1350021

2 (2013) 1350006

Carlen, P. L., see Serletis, D.

Carrette, E., see Vonck, K.

Cen, Z., Wei, J. \& Jiang, R., A gray-box neural network-based model identification and fault estimation scheme for nonlinear dynamic systems

Chakraborty, C., see Martis, R. J.

Chen, Y.-Y., see Liu, C.

Christodoulou, M., see Boutalis, Y.

Chua, C. K., see Martis, R. J.

Cichocki, A., see Cong, F.

Cona, F. \& Ursino, M., A multi-layer neural-mass model for learning sequences using theta/gamma oscillations

$1(2013) 1250030$

1 (2013) 1250033

1 (2013) 1250030

6 (2013) 1350027

1 (2013) 1250034

5 (2013) 1350022

$4(2013) 1350018$

1 (2013) 1250031

$1(2013) 1250033$

1 (2013) 1250034

6 (2013) 1350025

4 (2013) 1350014

4 (2013) 1350017

5 (2013) 1350022

5 (2013) 1350023

2 (2013) 1350006

$3(2013) 1250036$

Cong, F., Phan, A.-H., Astikainen, P., Zhao, Q., Wu, Q., Hietanen, J. K., Ristaniemi, T. \& Cichocki, A., Multi-domain feature extraction for small event-related potentials through nonnegative multi-way array decomposition from low dense array EEG 
Da Silva, F. L., see Koppert, M.

1 (2013) 1250032

Dauwe, I., see Mollet, L.

Dauwe, I., see Vonck, K.

6 (2013) 1350027

De Herdt, V., see Mollet, L.

1 (2013) 1250034

De Herdt, V., see Vonck, K.

6 (2013) 1350027

1 (2013) 1250034

Delbeke, J., see Mollet, L.

6 (2013) 1350027

del Campo, F., see Álvarez, D.

5 (2013) 1350020

Deng, B., see Liu, C.

Denham, S. L., see Dura-Bernal, S.

Deshpande, G., see Rangaprakash, D.

Dura-Bernal, S., Garreau, G., Georgiou, J., Andreou, A. G., Denham, S. L. \& Wennekers, T., Multimodal integration of micro-doppler sonar and auditory signals for behavior classification with convolutional networks

4 (2013) 1350017

5 (2013) 1350021

2 (2013) 1350003

El Tahry, R., see Mollet, L.

Enderle, J. D. \& Sierra, D. A., A new linear muscle fiber model for neural control of saccades

Fernandes, C. M., see Herrera, L. J.

García-Laencina, P. J., see Rodríguez-Bermúdez, G.

Garreau, G., see Dura-Bernal, S.

Garrido, J. A., see Tolu, S.

Gemignani, A., see Piaggi, P.

Gentili, C., see Piaggi, P.

Georgiou, J., see Dura-Bernal, S.

Glos, M., see Álvarez, D.

Goossens, L., see Vonck, K.

Grimonprez, A., see Mollet, L.

Guillen, A., see Herrera, L. J.

Guiot, C., see Stura, I.

Handjaras, G., see Piaggi, P.

He, W., see Li, G.

Herrera, L. J., Fernandes, C. M., Mora, A. M., Migotina, D., Largo, R., Guillen, A. \& Rosa, A. C., Combination of heterogeneous EEG feature extraction methods and stacked sequential learning for sleep stage classification

Hietanen, J. K., see Cong, F.

Hong, K., see Li, J.

Hong, T. J., see Acharya, U. R.

Horki, P., see Müller-Putz, G. R.

Hornero, R., see Álvarez, D.

Hsu, W.-Y., Application of quantum-behaved particle swarm optimization to motor imagery EEG classification

Hsu, W.-Y., Single-trial motor imagery classification using asymmetry ratio, phase relation, wavelet-based fractal, and their selected combination

5 (2013) 1350021

6 (2013) 1350027

2 (2013) 1350002

3 (2013) 1350012

4 (2013) 1350015

5 (2013) 1350021

3 (2013) 1350010

3 (2013) 1350011

3 (2013) 1350011

5 (2013) 1350021

5 (2013) 1350020

1 (2013) 1250034

6 (2013) 1350027

3 (2013) 1350012

2 (2013) 1350008

3 (2013) 1350011

4 (2013) 1350019

3 (2013) 1350012

2 (2013) 1350006

3 (2013) 1350013

3 (2013) 1350009

2 (2013) 1250037

5 (2013) 1350020

6 (2013) 1350026

2 (2013) 1350007

Hu, M. \& Liang, H., Perceptual suppression revealed by adaptive multi-scale entropy analysis of local field potential in monkey visual cortex

2 (2013) 1350005

2 (2013) 1350003

1 (2013) 1250030

6 (2013) 1350025

Jackson, G. D., see Badawy, R. A. B.

Jiang, R., see Cen, Z.

1 (2013) 1250032 
Kimiskidis, V. K., Kugiumtzis, D., Papagiannopoulos, S. \& Vlaikidis, N., Transcranial magnetic stimulation (TMS) modulates epileptiform discharges in patients with frontal lobe epilepsy: A preliminary EEG-TMS study

Klobassa, D. S., see Müller-Putz, G. R.

Kodogiannis, V. S., Amina, M. \& Petrounias, I., A clustering-based fuzzy wavelet neural network model for short-term load forecasting

Koppert, M., Kalitzin, S., Velis, D., Da Silva, F. L. \& Viergever, M. A., Reactive control of epileptiform discharges in realistic computational neuronal models with bistability

Krishnan, M. M. R., see Acharya, U. R.

Kugiumtzis, D., see Kimiskidis, V. K.

Landi, A., see Piaggi, P.

Largo, R., see Herrera, L. J.

Li, G., Ning, N., Ramanathan, K., He, W., Pan, L. \& Shi, L., Behind the magical numbers: Hierarchical chunking and the human working memory capacity

Li, H.-Y., see Liu, C.

Li, J., see Li, J.

Li, J., Liang, J., Zhao, Q., Li, J., Hong, K. \& Zhang, L., Design of assistive wheelchair system directly steered by human thoughts

Li, X., see Wang, Y.

Liang, H., see Hu, M.

Liang, J., see Li, J.

Lightbody, G., see Temko, A.

Liu, C., Wang, J., Chen, Y.-Y., Deng, B., Wei, X.-L. \& Li, H.-Y., Closed-loop control of the thalamocortical relay neuron's parkinsonian state based on slow variable

Lopez-Gordo, M. A. \& Pelayo, F., A binary phase-shift keying receiver for the detection of attention to human speech

Luque, N. R., see Tolu, S.

Müller-Putz, G. R., Pokorny, C., Klobassa, D. S. \& Horki, P., A single-switch BCI based on passive and imagined movements: Toward restoring communication in minimally conscious patients

Macdonell, R. A. L., see Badawy, R. A. B.

Mandana, K. M., see Martis, R. J.

Marcos, J. V., see Álvarez, D.

Marnane, W., see Temko, A.

Martens, H. C. F., see Cappaert, N. L. M.

Martis, R. J., Acharya, U. R., Min, L. C., Mandana, K. M., Ray, A. K. \& Chakraborty, C., Application of higher order cumulant features for cardiac health diagnosis using ECG signals

Martis, R. J., Acharya, U. R., Tan, J. H., Petznick, A., Tong, L., Chua, C. K. \& Ng, E. Y. K., Application of intrinsic time-scale decomposition (ITD) to EEG signals for automated seizure prediction

Martis, R. J., see Acharya, U. R.

Mauro, A., see Stura, I.

Meng, Q., see Wang, Y.

Menicucci, D., see Piaggi, P.

Meurs, A., see Mollet, L.

Meurs, A., see Vonck, K.

Miatton, M., see Vonck, K.

Migotina, D., see Herrera, L. J.
1 (2013) 1250035

2 (2013) 1250037

5 (2013) 1350024

1 (2013) 1250032

3 (2013) 1350009

1 (2013) 1250035

3 (2013) 1350011

3 (2013) 1350012

4 (2013) 1350019

4 (2013) 1350017

3 (2013) 1350013

3 (2013) 1350013

6 (2013) 1350028

2 (2013) 1350005

3 (2013) 1350013

4 (2013) 1350018

4 (2013) 1350017

4 (2013) 1350016

3 (2013) 1350010

2 (2013) 1250037

1 (2013) 1250030

4 (2013) 1350014

5 (2013) 1350020

4 (2013) 1350018

1 (2013) 1250031

4 (2013) 1350014

5 (2013) 1350023

3 (2013) 1350009

2 (2013) 1350008

6 (2013) 1350028

3 (2013) 1350011

6 (2013) 1350027

1 (2013) 1250034

1 (2013) 1250034

3 (2013) 1350012 
Min, L. C., see Acharya, U. R.

3 (2013) 1350009

Min, L. C., see Martis, R. J.

4 (2013) 1350014

Mollet, L., Raedt, R., Delbeke, J., El Tahry, R., Grimonprez, A., Dauwe, I., De Herdt, V., Meurs, A., Wadman, W., Boon, P. \& Vonck, K., Electrophysiological responses from vagus nerve stimulation in rats

Mora, A. M., see Herrera, L. J.

Ng, E. Y. K., see Martis, R. J.

Ning, N., see Li, G.

Olson, L. D. \& Perry, M. S., Localization of epileptic foci using multimodality neuroimaging

Pan, L., see Li, G.

Papagiannopoulos, S., see Kimiskidis, V. K.

Pelayo, F., see Lopez-Gordo, M. A.

Penzel, T., see Álvarez, D.

Perry, M. S., see Olson, L. D.

Petrounias, I., see Kodogiannis, V. S.

Petznick, A., see Martis, R. J.

Phan, A.-H., see Cong, F.

Piaggi, P., Menicucci, D., Gentili, C., Handjaras, G., Gemignani, A. \& Landi, A., Adaptive filtering and random variables coefficient for analyzing functional magnetic resonance imaging data

Pokorny, C., see Müller-Putz, G. R.

Priano, L., see Stura, I.

Raedt, R., see Mollet, L.

Raedt, R., see Vonck, K.

Ramanathan, K., see Li, G.

Ramekers, D., see Cappaert, N. L. M.

Rangaprakash, D., Hu, X. \& Deshpande, G., Phase synchronization in brain networks derived from correlation between probabilities of recurrences in functional MRI data

Ray, A. K., see Martis, R. J.

Ristaniemi, T., see Cong, F.

Roca-Dorda, J., see Rodríguez-Bermúdez, G.

Rodríguez-Bermúdez, G., García-Laencina, P. J. \& Roca-Dorda, J., Efficient automatic selection and combination of EEG features in least squares classifiers for motor imagery brain-computer interfaces

Ros, E., see Tolu, S.

Rosa, A. C., see Herrera, L. J.

Serletis, D., Carlen, P. L., Valiante, T. A. \& Bardakjian, B. L., Phase synchronization of neuronal noise in mouse hippocampal epileptiform dynamics

Shi, L., see Li, G.

Sierra, D. A., see Enderle, J. D.

Sprengers, M., see Vonck, K.

Stura, I., Priano, L., Mauro, A., Guiot, C. \& Venturino, E., Double-layered models can explain macro and micro structure of human sleep

Tan, J. H., see Martis, R. J.

Temko, A., Boylan, G., Marnane, W. \& Lightbody, G., Robust neonatal EEG seizure detection through adaptive background modeling

Theodoridis, D., see Boutalis, Y.

Thiery, E., see Vonck, K.

6 (2013) 1350027

3 (2013) 1350012

5 (2013) 1350023

4 (2013) 1350019

1 (2013) 1230001

4 (2013) 1350019

1 (2013) 1250035

4 (2013) 1350016

5 (2013) 1350020

1 (2013) 1230001

5 (2013) 1350024

5 (2013) 1350023

2 (2013) 1350006

3 (2013) 1350011

2 (2013) 1250037

2 (2013) 1350008

6 (2013) 1350027

1 (2013) 1250034

4 (2013) 1350019

1 (2013) 1250031

2 (2013) 1350003

4 (2013) 1350014

2 (2013) 1350006

4 (2013) 1350015

4 (2013) 1350015

3 (2013) 1350010

3 (2013) 1350012

1 (2013) 1250033

4 (2013) 1350019

2 (2013) 1350002

1 (2013) 1250034

2 (2013) 1350008

5 (2013) 1350023

4 (2013) 1350018

5 (2013) 1350022

1 (2013) 1250034 
Tolu, S., Vanegas, M., Garrido, J. A., Luque, N. R. \& Ros, E., Adaptive and predictive control of a simulated robot arm

Tong, L., see Martis, R. J.

Ursino, M., see Cona, F.

Valiante, T. A., see Serletis, D.

Van Roost, D., see Vonck, K.

Vanegas, M., see Tolu, S.

Velis, D., see Koppert, M.

Venturino, E., see Stura, I.

Viergever, M. A., see Koppert, M.

Vlaikidis, N., see Kimiskidis, V. K.

Vonck, K., Sprengers, M., Carrette, E., Dauwe, I., Miatton, M., Meurs, A., Goossens, L., De Herdt, V., Achten, R., Thiery, E., Raedt, R., Van Roost, D. \& Boon, P., A decade of experience with deep brain stimulation for patients with refractory medial temporal lobe epilepsy

Vonck, K., see Mollet, L.

Wadman, W. J., see Cappaert, N. L. M.

Wadman, W., see Mollet, L.

Wang, J., see Liu, C.

Wang, J., see Wang, Y.

Wang, Y., Zhou, W., Yuan, Q., Li, X., Meng, Q., Zhao, X. \& Wang, J., Comparison of ictal and interictal EEG signals using fractal features

Wei, J., see Cen, Z.

Wei, X.-L., see Liu, C.

Wei, Z. J., see Acharya, U. R.

Wennekers, T., see Dura-Bernal, S.

Wessel, N., see Álvarez, D.

Wu, Q., see Cong, F.

Yanti, R., see Acharya, U. R.

Yuan, Q., see Wang, Y.

Zhang, L., see Li, J.

Zhao, Q., see Cong, F.

Zhao, Q., see Li, J.

Zhao, X., see Wang, Y.

Zhou, W., see Wang, Y.

3 (2013) 1350010

5 (2013) 1350023

3 (2013) 1250036

1 (2013) 1250033

1 (2013) 1250034

3 (2013) 1350010

1 (2013) 1250032

2 (2013) 1350008

1 (2013) 1250032

1 (2013) 1250035

1 (2013) 1250034

6 (2013) 1350027

1 (2013) 1250031

6 (2013) 1350027

4 (2013) 1350017

6 (2013) 1350028

6 (2013) 1350028

6 (2013) 1350025

4 (2013) 1350017

3 (2013) 1350009

5 (2013) 1350021

5 (2013) 1350020

2 (2013) 1350006

3 (2013) 1350009

6 (2013) 1350028

3 (2013) 1350013

2 (2013) 1350006

3 (2013) 1350013

6 (2013) 1350028

6 (2013) 1350028 